\title{
TRABALHO DA ENFERMAGEM E DISTÚRBIO MUSCULOESQUELÉTICO: REVISÃO DAS PESQUISAS SOBRE O TEMA.
}

\author{
The nursing work and musculoskeletal disorders: a review of the \\ researches about the theme
}

\author{
Trabajo de la enfermería y disturbio musculoesquelético: revisión \\ de las investigaciones acerca del tema.
}

Tânia Solange Bosi de Souza Magnago ${ }^{1}$

Márcia Tereza Luz Lisboa²

Rosane Harter Griep ${ }^{3}$

\section{RESUMO}

Este estudo teve por objetivo identificar a produção brasileira de teses e disser tações sobre os distúrbios musculoesqueléticos em trabalhadores de enfermagem. As publicações foram pesquisadas no banco de teses e dissertações do Portal CAPES (Coordenação de Aperfeiçoamento de Pessoal de Nível Superior) e nos catálogos da ABEn (Associação Brasileira de Enfermagem), no período de 1978 a 2004. Foram selecionados os resumos de teses e dissertações de acordo com os seguintes critérios: desfecho "musculoesquelético" em população de trabalhadores de enfermagem. Dezoito estudos preencheram os critérios de inclusão. Destes, dois tiveram abordagem qualitativa; um, abordagem qualiquantitativa; e quinze, abordagem quantitativa. Os Cursos de Pós-graduação da Universidade de São Paulo e da Universidade Federal do Rio de Janeiro foram os que se destacaram em produções na temática em tela. Os trabalhos apontam tanto para o crescente adoecimento musculoesquelético dos trabalhadores de enfermagem quanto para as inadequadas condições de trabalho como fator de risco para esse adoecimento.

Palavras-chave: Transtornos Traumáticos Cumulativos. Saúde Ocupacional. Enfermagem. Condições de Trabalho.

\begin{abstract}
This study aimed to identify the Brazilian scientific production (thesis and dissertations) about musculoskeletal disorders in nursing workers. The publications were searched in the Banco de Teses e Dissertações (thesis and dissertations collection) of the Portal CAPES - Coordenação de Aperfeiçoamento de Pessoal de Nível Superior - (Brazilian Government Agency) and the catalogues of the Associação Brasileira de Enfermagem - ABEN (Brazilian Nursing Association). The period researched was 19782004. The criterion used to select the thesis and dissertations' abstract was the outcome "musculoskeletal" in nursing population. Eighteen studies matched the criterion. Regarding to the research approach, 2 of them presented a qualitative one, 1 quantitative -qualitative one; and 15 quantitative one. The majority of them were from pos-graduated courses of Universidade de São Paulo and Universidade Federal do Rio de Janeiro. The studies pointed out to the growing number of nursing workers who have became sick due to musculoskeletal disorders; as well as to the poor conditions of work as a risk factor for this.
\end{abstract}

Keywords: Cumulative Trauma Disorders. Occupational Health. Nursing. Working Conditions.

\section{Resumen}

Este estudio tuvo por objetivo identificar la producción brasileña de tesis y disertaciones acerca los disturbios musculoesqueléticos en trabajadores de enfermería. Las publicaciones han sido investigadas en el Banco de Tesis y Disertaciones del Portal CAPES - "Coordenação de Aperfeiçoamento de Pessoal de Nível Superior" y en los catálogos de la ABEn - "Associação Brasileira de Enfermagem", en el período de 1978 a 2004 . Fueron seleccionados los resúmenes de tesis y disertaciones de acuerdo con los siguientes criterios: el cierre "musculoesquelético" en población de trabajadores de enfermería. Dieciocho estudios cumplían los criterios de inclusión. De estos, dos tuvieron abordaje cualitativo, un cuali-cuantitativo y quince abordaje cuantitativo. Los cursos de Postgrado de la Universidad de São Paulo y de la Universidad Federal del Rio de Janeiro fueron los que se destacaron en producciones en la temática en discusión. Los trabajos señalan tanto para el hecho de adolecer musculoesquelético creciente de los trabajadores de enfermería cuanto para las inadecuadas condiciones de trabajo como factor de riesgo para ese adolecer.

Palabras clave: Transtornos de traumas acumulados. Salud labora. Enfermería. Condiciones de trabajo. 


\section{INTRODUCÃO}

0 presente estudo trata da revisão e da análise crítica da produção científica na área da saúde do trabalhador de enfermagem, no período de 1978 a 2004 . 0 objeto de estudo eleito foram os trabalhos que abordam a saúde do trabalhador de enfermagem com enfoque no tema: distúrbio musculoesquelético em trabalhadores de enfermagem.

Como fonte de referência, foram utilizados os Catálogos de Produção Científica da Enfermagem da Associação Brasileira de Enfermagem ${ }^{1,2}$ - CPCENF/ABEn e o Banco de Teses e Dissertações da Coordenação de Aperfeiçoamento de Pessoal de Nível Superior ${ }^{3}$ - CAPES.

A opção pelo estudo deve-se ao fato de que os distúrbios musculoesqueléticos estão entre as causas mais comuns de consulta médica e correspondem à terceira causa de afastamento do trabalho ${ }^{4}$. Na atualidade, sabe-se que 0 desenvolvimento de distúrbio musculoesquelético é multicausal, sendo imprescindível a avaliação dos fatores de risco a ele relacionado, direta ou indiretamente. Pesquisadores europeus e americanos apontam os aspectos psicossociais do trabalho (demandas do trabalho e controle do trabalhador sobre o processo de trabalho) como um importante fator a ser investigado no ambiente laboral.

Com este intuito, uma das autoras está desenvolvendo uma tese de doutorado no Núcleo de Pesquisa Enfermagem e Saúde do Trabalhador - NUPENST, do Departamento de Enfermagem de Saúde Pública, da Escola de Enfermagem Anna Nery (EEAN) da Universidade Federal do Rio de Janeiro (UFRJ), cuja questão da pesquisa é "existe associação entre altas demandas psicológicas no trabalho e baixo controle do trabalhador sobre a atividade laboral e desenvolvimento de distúrbios musculoesquelético em trabalhadores de enfermagem?"

Este estudo teve por objetivos: identificar a produção brasileira de teses e dissertações sobre os distúrbios musculoesqueléticos em trabalhadores de enfermagem; identificar os objetos de estudos abordados nessas pesquisas; e identificar quais temáticas não estão sendo abordadas no sentido de encontrar as lacunas no estado da arte desses estudos.

\section{TRABALHO E DISTÚRBIO MUSCULOESQUELÉTICO: UM BREVE HISTÓRICO}

Os distúrbios musculoesqueléticos, nas últimas décadas, têm se constituído um grande problema da saúde pública, principalmente para os países industrializados ${ }^{4}$. Na literatura mundial, são utilizadas várias terminologias para descrevêlos, como, por exemplo, lesões por esforço repetitivo (LER), doença osteomuscular relacionada ao trabalho (DORT), lesões por traumas cumulativos, lesões ou distúrbios musculoesqueléticos, entre outros.

Neste artigo será utilizado o termo distúrbio musculoesquelético, entendendo-o como uma síndrome relacionada ao trabalho e caracterizada por: dor, parestesia, sensação de peso e fadiga, de aparecimento insidioso, geralmente nos membros superiores, mas podendo ocorrer em membros inferiores ${ }^{4}$.

Esse distúrbio tem início insidioso, e muitas vezes o trabalhador não se dá conta do seu acometimento precocemente. Por ser intermitente, de curta duração e de leve intensidade, a sintomatologia pode passar por cansaço passageiro ou 'mau jeito'. Caso não sejam tomadas medidas de tratamento e prevenção de novos episódios, aos poucos, os sintomas se intensificam e aparecem de forma espontânea e contínua, comprometendo a capacidade funcional do trabalhador, tanto no trabalho quanto na vida familiar ${ }^{4}$.

0 surgimento de distúrbios musculoesqueléticos no trabalho, apesar de ter se intensificado nas últimas décadas, não é contemporâneo. Encontra-se descrito na literatura desde a Antiguidade. Hipócrates (460 - 375 a.C.), em sua obra Epidemia, descreveu o caso de um trabalhador que desenvolvera paralisia na mão após torcer ramagens por tempo prolongado ${ }^{4}$. Desde Hipócrates, é possível observar algumas referências sobre a associação entre trabalho e a saúdedoença ${ }^{5}$.

No entanto, somente em 1700 é que o médico italiano Bernardino Ramazzini (1613 - 1714), após observar doenças que ocorreram em trabalhadores de mais de 50 ocupações, dentre elas a dos escrivões, introduz na anamnese a pergunta: qual é a sua ocupação? Tal argüição tem possibilitado ao profissional de saúde chegar às causas do adoecimento e a estabelecer um tratamento mais eficiente e eficaz ${ }^{5}$.

Nas últimas décadas, é crescente a discussão em torno das doenças osteomusculares relacionadas ao trabalho, principalmente devido ao aumento da prevalência desta patologia nas mais diversas ocupações. Na década de 1950, no Japão, observou-se um aumento na prevalência de trabalhadores acometidos por uma doença chamada 'síndrome cervicobraquial'. Na Austrália, ao final da década de 1970, houve uma explosão de casos, considerados como 'neurose ocupacional'. Nas décadas de 1980 e 1990 houve o aparecimento de epidemias da doença tanto nos EUA como na Suécia ${ }^{6}$.

No Brasil, as lesões por esforços repetitivos/distúrbios osteomusculares relacionados ao trabalho (LER/DORT) começam a ser identificadas na década de 1970. Em 1973, essa patologia foi identificada em lavadeiras, limpadoras e engomadeiras, recomendando-se que fossem feitas pausas de trabalho para trabalhadores que desenvolviam atividades intensas com as mãos ${ }^{4}$.

Em 1986, o Instituto Nacional de Assistência Médica da Previdência Social (INAMPS) publicou uma circular orientando 
as superintendências para que reconhecessem a tenossinovite como doença do trabalho, sendo que os digitadores, mecanógrafos, datilógrafos, pianistas, caixas, grampeadores, costureiras e lavadeiras, entre outras categorias, eram as que freqüentemente se expunham à sobrecarga tendinosa e inserção muscular, ocasionando tal patologia.

Em 1991, o Instituto Nacional de Seguridade Social (INSS) publicou as Normas Técnicas para Avaliação de Incapacidade. Nelas estão contidos os critérios de diagnóstico e tratamento e a descrição de casos de LER em diversas ocupações: digitadores, operadores de máquinas/computadores, telefonistas, pedreiros, ajudantes de laboratório, técnicos administrativos, entre outros.

Em 1993, o INSS publicou uma revisão das Normas sobre LER e ampliou o seu conceito, reconhecendo na sua etiologia, além dos fatores biomecânicos, os relacionados à organização do trabalho. Importante ressaltar que, pela Portaria 3.214, de 8 de junho de 1978, foram aprovadas as Normas Regulamentadoras (NRs), relativas à Segurança e Medicina do Trabalho, no Brasil. Atualmente, temos publicadas 33 NRs. Destas, pelo menos duas trazem avanços importantes em relação ao tema estudado nesta pesquisa. A NR 17 traz aspectos da ergonomia e visa "estabelecer parâmetros que permitam a adaptação das condições de trabalho às características psicofisiológicas dos trabalhadores, de modo a proporcionar um máximo de conforto, segurança e desempenho eficiente" 7:1. Para condições de trabalho estão incluídos aspectos que dizem respeito ao levantamento, transporte e descarga de materiais, ao mobiliário, aos equipamentos e às condições ambientais do posto de trabalho e à própria organização do trabalho ${ }^{7}$. Entretanto, nessa norma não há nenhuma menção quanto ao controle ou a aspectos destinados a aumentar a participação do trabalhador nas definições dos processos laborais.

Em novembro de 2005, foi aprovada a NR 32 (Portaria n 485/05) que tem por finalidade "estabelecer diretrizes básicas para a implementação de medidas de proteção à segurança $\mathrm{e}$ à saúde dos trabalhadores dos serviços de saúde..." "7:1. Lançando um olhar crítico sobre esta NR, percebe-se que ela contemplou diretrizes somente sobre alguns aspectos na implementação das medidas de proteção contra os riscos, pois enfocou os riscos biológicos, os químicos e os das radiações ionizantes, ficando algumas questões sobre organização dos postos de trabalho como, por exemplo, deslocamentos, esforços adicionais, movimentação e transporte de pacientes e materiais, descritas apenas nas Disposições Gerais da NR. Estas questões, pouco discutidas na NR, podem ser fatores decisivos no adoecimento de muitos trabalhadores de enfermagem.

Algumas pesquisas apontam que as sintomatologias musculoesqueléticas referidas por trabalhadores de enfermagem estão diretamente relacionadas a atividades como, por exemplo, movimentação e transporte de pacientes; banho de leito e percorrer grandes distâncias durante 0 desenvolvimento das tarefas $8,9,10,11$. Como situações predisponentes ao desenvolvimento de distúrbios musculoesqueléticos, são evidenciadas a fadiga e as condições de trabalho ${ }^{12,13}$, e as condições organizacionais e ergonômicas $^{14}$.

De acordo com a Instrução Normativa/INSS n 98/2003, os fatores de risco para distúrbios musculoesqueléticos podem ser: grau de adequação do posto de trabalho à zona de atenção e à visão; frio, vibrações e pressões locais sobre os tecidos; posturas inadequadas; carga osteomuscular; carga estática; invariabilidade da tarefa; exigências cognitivas e fatores organizacionais e psicossociais ligados ao trabalho.

\section{METODOLOGIA}

Trata-se de uma pesquisa bibliográfica, realizada em maio de 2006, que apresenta a revisão e análise crítica de teses e dissertações acerca da temática dos distúrbios musculoesqueléticos em trabalhadores de enfermagem.

A coleta de dados teve como fontes principais os resumos de teses de doutorado e dissertações de mestrado produzidas no período de 1978 a 2004 e publicadas nos Catálogos da ABEn e no Banco de Teses e Dissertações da CAPES.

Para as publicações nos Catálogos da ABEn, realizamos a busca nos descritores: saúde ocupacional, coluna vertebral, condições de trabalho, dor lombar, enfermagem ocupacional, risco ocupacional, trabalhadores e trabalho. Para a busca no Portal CAPES, utilizamos os descritores: saúde do trabalhador, saúde ocupacional, enfermagem, musculoesquelético e osteomuscular.

Selecionamos, inicialmente, todos os trabalhos que apresentavam no título a temática proposta neste artigo. Nos títulos que não continham as palavras distúrbios musculoesquelético ou osteomuscular e enfermagem, mas que davam indícios de que essa temática poderia ser abordada ao longo do trabalho, realizamos a leitura do resumo para que não se perdesse nenhum estudo.

Para análise dos dados, elaboramos um formulário contendo as seguintes categorias: autor, ano de defesa, período e local da pesquisa, objeto de estudo, problema de investigação, objetivos, marco teórico, método ou tipo de estudo, sujeitos, estratégia de coleta dos dados, tratamento dos dados e principais resultados. Após a seleção dos resumos, fizemos a leitura crítica dos mesmos, fazendo um mapeamento dessas produções e destacando as principais categorias (Tabela1). 


\section{APRESENTAÇÃO DOS RESULTADOS}

As teses e dissertações encontradas e identificadas, de acordo com os descritores referidos, estão sumarizadas na tabela a seguir.

Tabela 1 - Teses e dissertações publicadas nos catálogos da ABEn e do Banco do Portal Capes, no período de 1978 a 2004 , Brasil.

\begin{tabular}{|c|c|c|c|c|}
\hline AutorReferência & Ano & $\begin{array}{l}\text { Escola } \\
\text { Enf }\end{array}$ & $\begin{array}{l}\text { Método/ } \\
\text { Técnica de coleta/ } \\
\text { População } \\
\end{array}$ & Resultados \\
\hline Mauro $^{12}$ & 1977 & UFRJ & $\begin{array}{l}\text { Questionário. Análise ergonômica. } \\
\text { Docentes de enfermagem. }\end{array}$ & $\begin{array}{l}\text { Os professores de enfermagem se encontram } \\
\text { em condições que caracterizam a fadiga } \\
\text { imprópria no trabalho. }\end{array}$ \\
\hline Alexandre ${ }^{13}$ & 1987 & USP & $\begin{array}{l}\text { Observação nos procedimentos de } \\
\text { transferência para leito/maca e vice-versa. } \\
360 \text { trabalhadores de enfermagem de } \\
\text { unidade de internação. }\end{array}$ & $\begin{array}{l}\text { Os trabalhadores realizam os procedimentos sob } \\
\text { condições desfavoráveis, equipe insuficiente e } \\
\text { equipamentos inadequados e sem manutenção. } \\
\text { Procedimento realizado por atendentes de } \\
\text { enfermagem; o método utilizado depende das } \\
\text { condições do paciente e do número de pessoas } \\
\text { disponíveis no momento. }\end{array}$ \\
\hline
\end{tabular}

Alexandre $^{8} \quad 1993 \quad$ USP

Marziale $^{15}$

Zeitoune $^{16}$

Rocha $^{17}$

UFMG

Entrevista. Observação ergonômica do ambiente de trabalho.Trabalhadores de enfermagem de unidade de internação em clínica médica.

Amarante $^{9}$

1999

USP
Entrevista e avaliação ergonômica.75 trabalhadores de enfermagem de um hospital governamental.

Não especifica 0 método no resumo. Trabalhadores de enfermagem de unidade de internação cardiologia.

Questionário ergonômico. Cinematografia. 11 auxiliares de enfermagem de sala de vacinas em um centro de saúde.
Auxiliares e atendentes de enfermagem são mais acometidos por cervicolombalgias. As dores nas costas são produzidas principalmente por movimentação e transporte de pacientes, manutenção de posturas inadequadas e estáticas, mobiliários e equipamentos inadequados.

Problemas identificados: dupla jornada, baixos salários, relacionais, inadequação dos postos de trabalho e níveis de iluminação e temperatura inadequados. Elevada freqüência de absenteísmodoença.

Profissionais do sexo feminino; tempo médio de 11 a 15 anos de trabalho no setor. Dor nas costas em $90 \%$ dos trabalhados trabalhadores. Fator de risco para dor lombar: postura adotada para o procedimento. Os episódios de dor forte a muito forte ocorriam há mais de um ano, com freqüência à noite e durante $o$ trabalho na vacinação.

$89 \%$ dos trabalhadores referiram algum tipo de algia vertebral. $71,4 \%$ dor lombar. Correlação significativa $(p<0,05)$ entre dor nas costas, dupla jornada de trabalho, mudança de decúbito em colchão d'água e higiene íntima. A observação evidenciou: incompatibilidade entre os espaços existentes entre os leitos para atender a necessidade de movimentos corporais dos trabalhadores e manipulação de utensílios e aparelhos nestes locais. Inadequação do mobiliário, utensílios e equipamentos quanto às dimensões, alturas das superfícies, peso e condições de funcionamento. Falta de avaliação do esforço físico por tarefa; ausência de padronização das técnicas, posturas inadequadas aumentam a sobrecarga física e o índice de dor nas costas.

Ritmo de trabalho intenso. Tarefas que exigem rapidez de raciocínio e precisão; freqüentes deslocamentos. Os enfermeiros estão expostos a cargas físicas, químicas, mecânicas, biológicas e psíquicas. 


\begin{tabular}{|c|c|c|c|c|}
\hline Autor Referência & Ano & $\begin{array}{l}\text { Escola } \\
\text { Enf }\end{array}$ & $\begin{array}{c}\text { Método/ } \\
\text { Técnica de coleta/ } \\
\text { População }\end{array}$ & Resultados \\
\hline Guedes $^{10}$ & 2000 & UERJ & $\begin{array}{l}\text { Quantiqualitativa. Pesquisa } \\
\text { ergonômica. Análise de discurso. } \\
\text { Questionário auto-aplicativo. Diagrama } \\
\text { de registro da localização da dor } \\
\text { musculoesquelética. } 19 \text { auxiliares de } \\
\text { enfermagem de unidade ortopédica. }\end{array}$ & $\begin{array}{l}68,42 \% \text { sexo feminino. Idade média } 37,8 \text { anos. } \\
\text { Escolaridade no nível médio ( } 47,35 \%) \text {, média de trabalho } \\
\text { de } 9,6 \text { anos e no setor } 5,5 \text { anos, com renda mensal de } \\
9 \text { salários. Horas livres são dedicadas a atividades } \\
\text { domésticas, não realizam "check-up" nem exames } \\
\text { periódicos. Posição em pé é freqüente }(84,25 \%) \text {, com } \\
\text { muito dispêndio de energia. Maior esforço físico no } \\
\text { transporte e mobilização dos pacientes. Necessidade } \\
\text { de melhoria nas condições de trabalho. Alta incidência } \\
\text { de sensação dolorosa relacionada a problemas } \\
\text { osteomusculares. A maioria não reconhece a relação da } \\
\text { dor com problemas de saúde. }\end{array}$ \\
\hline
\end{tabular}

\begin{tabular}{|c|c|c|c|}
\hline Lemos $^{18}$ & 2001 & UFSC & $\begin{array}{l}\text { Epidemiológico transversal. } \\
\text { Anamnese Clínico-ocupacional. Escala } \\
\text { de avaliação psíquica. } 81 \text { trabalhadores } \\
\text { de enfermagem com diagnóstico de } \\
\text { DORT. }\end{array}$ \\
\hline
\end{tabular}

Fontes $^{19}$

Radovanovic ${ }^{20}$

2002

Góes ${ }^{11}$

2002

Moreira $^{14}$

Rezende 21

Varela ${ }^{22}$

2004

USP

Murofuse 23

Goulart24
UFBa

Quantitativo, retrospectivo e descritivo. Enfermeiros de um hospital de reabilitação.

JEC Ergonomia. Enfermeiros.
Ergonomia. Avaliação do ambiente de trabalho e mobiliário. Observação da postura corporal. Formulário.

25 trabalhadores de enfermagem de unidade de internação.

UFRJ Documental. Formulário.

Levantamento das licenças médicas. 43 trabalhadores enfermagem.

Estudo seccional, descritivo. Questionário. Entrevista.85 auxiliares de enfermagem.

Qualitativo. Materialismo históricodialético. Entrevista semi-estruturada. Observação. 25 trabalhadores de enfermagem.

cumental. Quantitativo, descritivo. Levantamento dos atendimentos aos Trabalhadores. 4.307 trabalhadores de enfermagem.

$85 \%$ dos trabalhadores referiram, pelo menos, um episódio de dor nos últimos seis meses. Intensidade da dor: leve a insuportável. A dor provoca alterações no estado emocional. Todas as categorias e em todas as faixas etárias percebem no trabalho as cargas psíquicas, mas descartam a existência de fatores predisponentes.

Principal causa de absenteísmo: osteomuscular (36\%). Déficit na participação do enfermeiro na contribuição da ergonomia desde a concepção, conscientização até correção de fatores de risco.

Elaboração de um instrumento para avaliar os riscos ergonômicos durante os procedimentos de movimentação e transferência de pacientes. Os resultados indicaram que o instrumento poderá contribuir para um melhor planejamento da assistência de enfermagem.

Ambiente: espaços restritos no banheiro, mobiliários com altura fixa incompatível que provocam a adoção de posturas inadequadas. Postura: as atividades que mais causam adoção de postura inadequada e por maior tempo são a mudança de decúbito (8'33") e 0 banho de leito (4'59"). $100 \%$ dos enfermeiros e $97,70 \%$ dos auxiliares relatam dor na coluna (lombar e cervical).

Principais fatores de risco para DORT são os de natureza organizacional e ergonômica. As DORTs foram atribuídas às condições inadequadas de trabalho.

$21,57 \%$ referiram dorsalgia não especificada e dor lombar baixa. A maioria dos auxiliares de enfermagem não identifica os riscos físicos no trabalho.

Posturas incorretas, manipulação de peso excessivo e repetitividade resultam na redução ou impedimento do exercício das atividades ocupacionais. As mudanças são insuficientes com relação à garantia de condições dignas de trabalho. Falta de orientações relativas às lesões por esforços repetitivos.

$11,85 \%$ doenças do sistema osteomuscular (segunda maior causa). $55,7 \%$ possuem vínculo temporário de trabalho.

Significância estatística associada à dor nas costas e postura inadequada no trabalho. 


\section{Referências}

1.Associação Brasileira de Enfermagem- ABEn. Centro de Estudos e Pesquisas em Enfermagem. Informações sobre pesquisas e pesquisadores em enfermagem: 1979-2000. Brasília (DF): ABEn; 2001.

2.Associação Brasileira de Enfermagem- ABEn. Centro de Estudos e Pesquisas em Enfermagem. Informações sobre pesquisas e pesquisadores em enfermagem: 2001- 2004. [CD-ROM]. Brasília (DF): ABEn; 2001.

3.Coordenação de Aperfeiçoamento de Pessoal de Nível Superior CAPES. Banco de Teses:1987 - 2004. [on-line] [citado maio 2006]. Disponível em: http://servicos.capes.gov.br/capesdw/.

4.Maemo M, Carmo JC. Saúde do trabalhador no SUS: aprender com o passado, trabalhar para o presente, construir o futuro. São Paulo (SP): Hucitec; 2005.

5.Mendes R, organizador. Patologia do trabalho. Rio de Janeiro (RJ): Atheneu; 1995.

6.Pinheiro FA, Tróccoli BT, Paz MGT. Aspectos psicossociais dos distúrbios osteomusculares - DORT/LER relacionados ao trabalho. In: Mendes AM, Borges LO, Ferreira MCF, organizadores. Trabalho em transição, saúde em risco. Brasília (DF): Universidade de Brasília; 2002. p 66-85.

7.Ministério do Trabalho e Emprego (BR). Normas regulamentadoras. [on-line] [citado 11 set 2006]. Disponível em: http://www.mte.gov.br 8.Alexandre NMC. Contribuição ao estudo da cervicodorsolombalgia em profissionais de enfermagem. [tese de doutorado]. Ribeirão Preto (SP): Escola de Enfermagem /USP; 1993.

9.Amarante ST. Análise das condições ergonômicas do trabalho das enfermeiras de Centro Cirúrgico. [dissertação de mestrado]. São Paulo (SP): Escola de Enfermagem/USP; 1999.

10.Guedes EM. Distúrbios osteomusculares e o trabalho de enfermagem hospitalar: estudo com auxiliares de enfermagem em unidade de ortopedia. Rio de Janeiro (RJ): Centro Biomédico/Faculdade de Enfermagem/ UERJ; 2000.

11.Góes GC. Posturas adotadas e a ocorrência de cervicodorsolombalgia nos trabalhadores de enfermagem em uma unidade de internação. [dissertação de mestrado]. São Paulo (SP): Escola de Enfermagem/USP; 2002.

12.Mauro MIC. A fadiga e trabalho docente de enfermagem. [tese de livre docência]. Rio de Janeiro (RJ): Escola de Enfermagem Nery/ UFRJ; 1977.

13.Alexandre NMC. Avaliação de determinados aspectos ergonômicos no transporte de pacientes. [dissertação de mestrado]. Ribeirão Preto (SP): Escola de Enfermagem /USP; 1987.

14.Moreira AMR. Fatores de risco dos distúrbios osteomusculares relacionados ao trabalho de enfermagem: cenários e proposta de mudança. [dissertação de mestrado]. Rio de Janeiro (RJ): Centro Biomédico/Faculdade de Enfermagem/UERJ; 2003.

15.Marziale, Maria Helena Palucci. Condições ergonômicas da situação de trabalho do pessoal de enfermagem, em uma unidade de internação hospitalar. [tese de doutorado]. Ribeirão Preto (SP): Escola de Enfermagem/USP; 1995.
16.Zeitoune RCG. Desconfor to lombar e as variáveis cinemáticas da postura do profissional de enfermagem. [tese de doutorado]. Rio de Janeiro (RJ): Escola de Enfermagem Anna Nery/UFRJ; 1996.

17. Rocha AM. Fatores ergonômicos e traumáticos envolvidos na ocorrência de dor nas costas em trabalhadores de enfermagem. [dissertação de mestrado]. Belo Horizonte (MG): Escola de Enfermagem/UFMG; 1997.

18. Lemos JC. Avaliação da carga psíquica nos distúrbios osteomusculares relacionados ao trabalho (DORT) em trabalhadores de enfermagem. [dissertação de mestrado]. Florianópolis (SC): Faculdade de Psicologia/UFSC; 2001.

19.Fontes AM. Absenteísmo e os aspectos ergonômicos do trabalho na enfermagem. [dissertação de mestrado]. Salvador (BA): Escola de Enfermagem/UFBa; 2001.

20.Radovanovic CAT. Desenvolvimento e validação de um instrumento para avaliar a movimentação e transferência de pacientes: uma abordagem ergonômica. [dissertação de mestrado]. Campinas (SP): Faculdade de Ciências Médicas/UEC; 2002.

21. Rezende MP. Agravos à saúde de auxiliares de enfermagem resultantes da exposição ocupacional aos riscos físicos. [dissertação de mestrado]. Ribeirão Preto (SP): Escola de Enfermagem de Ribeirão Preto/USP; 2003.

22.Varela CDC. Trabalhadoras de enfermagem com lesão por esforço repetitivo- LER. [dissertação de mestrado]. Salvador (BA): Escola de Enfermagem/UFBa; 2004.

23.Murofuse MT. Adoecimento dos trabalhadores de enfermagem da Fundação Hospitalar do Estado de Minas Gerais: reflexo das mudanças no mundo do trabalho. [tese de doutorado]. Ribeirão Preto (SP): Escola de Enfermagem /USP; 2004.

24.Goulart LQ. Trabalhadores de hospital: riscos à saúde com especial ênfase no trabalho em turnos e noturno. [dissertação de mestrado]. São Paulo (SP): Faculdade de Saúde Pública/USP; 2004.

25.Froemming MB. Proposta de melhoramentos do equipamento de transferência visando o confor to do idoso e do cuidador. [dissertação de mestrado]. Santa Maria (RS): Faculdade de Engenharia da Produção/ UFSM; 2004.

26.Beck CLC, Gonzáles RMB, Stekel LMC, Donaduzzi JC. 0 trabalho da enfermagem em unidades críticas e sua repercussão sobre a saúde dos trabalhadores. Esc Anna Nery Rev Enferm 2006 ago; 10 (2): 221-27. 